\title{
Covariant Baryon Charge Radii and Magnetic Moments in a
} Chiral Constituent Quark Model*

\author{
K. Berger, R. F. Wagenbrunn, W. Plessas \\ Theoretische Physik, Karl-Franzens-Universität, Graz, Austria
}

\begin{abstract}
The charge radii and magnetic moments of all the light and strange baryons are investigated within the framework of a constituent quark model based on Goldstone-boson-exchange dynamics. Following the point-form approach to relativistic quantum mechanics, the calculations are performed in a manifestly covariant manner. Relativistic (boost) effects have a sizeable influence on the results. The direct predictions of the constituent quark model are found to fall remarkably close to the available experimental data.
\end{abstract}

PACS numbers: 12.39.Ki, 13.40.Em, 14.20.Dh, 14.20.Jn

Keywords: Electric radii; Magnetic Moments; Constituent quark model; Point-form quantum mechanics

\section{INTRODUCTION}

The charge radii and magnetic moments, as measures for the distribution of charge and magnetisation, represent important observables in hadronic physics. While quantum chromodynamics (QCD) is accepted as the fundamental theory of strong interactions, one is still lacking its direct predictions for this kind of observables. The reason is simply that QCD cannot (yet) be solved - accurately enough - in the low-energy domain of hadron ground-state properties, even though one has recently made progress in deducing hadron masses and also has obtained first results for hadron charge radii and magnetic moments from lattice QCD [1, 2, 3] (for a review see, e.g., [4]).

In view of the difficulties of solving QCD at low energies one has developed effective (field) theories and/or effective models. A promising approach is offered by constituent quark models (CQMs). Modern CQMs can be constructed so as to include the relevant properties of QCD in the low-energy regime, notably the consequences of the spontaneous breaking of chiral symmetry $(\mathrm{SB} \chi \mathrm{S})$. At the same time the requirements of special relativity can be incorporated by making the theory Poincaré-invariant.

Some time ago the Graz group suggested an interesting new type of CQM [5]. Its dynamics was motivated by the idea that at low energies the dominant QCD degrees of freedom are furnished by constituent quarks and Goldstone bosons $[\underline{6}$. The so-called Goldstone-boson-exchange (GBE) CQM for baryons relies on the Hamilton operator 5 ]

$$
\hat{H}=\sum_{i=1}^{3} \sqrt{\hat{\vec{p}}_{i}^{2}+\hat{m}_{i}^{2}}+\sum_{i<j=1}^{3}\left[\hat{V}_{\text {conf }}(i j)+\hat{V}_{h f}(i j)\right]
$$

which is defined on the $\{Q Q Q\}$ Hilbert space $\mathcal{H}$. The first term represents the relativistic kinetic-energy operator, where $\hat{\vec{p}}_{i}$ and $\hat{m}_{i}$ are the three-momentum and mass operators of the constituent quarks, respectively. The confinement potential $\hat{V}_{\text {conf }}(i j)$ is taken in linear form with a strength in accordance with the string tension of QCD. The hyperfine interaction $\hat{V}_{h f}(i j)$ consists of the spin-spin part of the exchange of octet and singlet pseudoscalar bosons (mesons) and produces a specific spin-flavor dependence [5, 7]. With this hyperfine interaction the level orderings of positiveand negative-parity states in the $N$ and $\Lambda$ spectra can be reproduced simultaneously in agreement with experiment (the detailed spectra of the GBE CQM may be found in refs. [5, 7]). The Hamiltonian of eq. (11) also lends itself to an invariant mass operator in relativistic Hamiltonian dynamics (RHD) [8]. This turns out to be very important for obtaining relativistic results of all kind of observables in hadron reactions.

Beyond spectroscopy the GBE CQM has in the meantime been put to testing its performance with regard to the electroweak structure of the nucleons. Specifically one has produced its predictions for the nucleon elastic electromagnetic [9] and axial form factors [10]. Working in the framework of the point-form version of RHD [11, 12] one has obtained covariant results for all elastic observables relating to nucleon electroweak form factors [13]. The direct predictions of the GBE CQM have been found to be in close agreement with all existing data in the low-momentum transfer domain (up to a few $\mathrm{GeV}^{2}$ ).

\footnotetext{
* This work was supported by the Austrian Science Fund, project no. P14806-TPH.
} 
In this work we present first covariant results of the GBE CQM for charge radii and magnetic moments of all the octet and decuplet baryon ground states. We again apply the point-form version of RHD. Whenever possible the theoretical predictions are compared to experiments.

\section{FORMALISM OF THE POINT FORM}

The point form was already defined in 1949 by Dirac [11] when he studied RHD with regard to the (smallest) stability groups of Poincaré generators of an interacting system. It is characterized by the fact that only the fourmomentum operator $\hat{P}^{\mu}$ is affected by interactions. All other generators of the Poincaré group remain interaction-free. As a result the spatial rotations and, most importantly, the Lorentz boosts are purely kinematic. Consequently, the theory is manifestly covariant.

In the point form the problem of solving the Hamiltonian of eq. (1) is completely equivalent to the solution of the eigenvalue problem of the mass operator $\hat{M}=\hat{M}_{\text {free }}+\hat{M}_{\text {int }}$, where the interaction $\hat{M}_{\text {int }}$ is added to the free mass operator $\hat{M}_{\text {free }}=\sqrt{\hat{P}_{\text {free }}^{\mu} \hat{P}_{\text {free }, \mu}}$ according to the Bakamjian-Thomas (BT) construction [14]. Correspondingly the four-momentum operator gets split into a free part $\hat{P}_{f r e e}^{\mu}$ and an interaction part $\hat{P}_{i n t}^{\mu}$

$$
\hat{P}^{\mu}=\hat{P}_{\text {free }}^{\mu}+\hat{P}_{i n t}^{\mu}=\hat{M} \hat{V}^{\mu}=\left(\hat{M}_{\text {free }}+\hat{M}_{\text {int }}\right) \hat{V}^{\mu}
$$

It is a peculiarity of the point form that in the BT construction the four-velocity remains interaction-free: $\hat{V}^{\mu}=\hat{V}_{f r e e}^{\mu}$. When solving the eigenvalue equation of the interacting mass operator

$$
\hat{M}\left|\Psi_{B}\right\rangle=M_{B}\left|\Psi_{B}\right\rangle
$$

one obtains the eigenvalues $M_{B}$ and the eigenstates $\left|\Psi_{B}\right\rangle$ for baryon $B$ on the Hilbert space $\mathcal{H}$. Due to the commutation relations of the Poincaré algebra the $\left|\Psi_{B}\right\rangle$ are simultaneous eigenstates of $\hat{P}^{\mu}(\mu=0,1,2,3)$ and thus also of the Hamiltonian $\hat{H}$ of eq. (1). Furthermore, they are simultaneously eigenstates of the four-velocity operator $\hat{V}^{\mu}$ and evidently of the total-angular-momentum operator $\hat{J}$ and its z-component $\hat{\Sigma}$. Therefore we shall subsequently characterize them by the corresponding eigenvalues and express them as $\left|v_{B}, M_{B}, J, \Sigma\right\rangle$.

The $\{Q Q Q\}$ Hilbert space $\mathcal{H}$ is spanned by the free states

$$
\left|p_{1}, \sigma_{1} ; p_{2}, \sigma_{2} ; p_{3}, \sigma_{3}\right\rangle=\left|p_{1}, \sigma_{1}\right\rangle \otimes\left|p_{2}, \sigma_{2}\right\rangle \otimes\left|p_{3}, \sigma_{3}\right\rangle
$$

which are direct products of free single-particle states $\left|p_{i}, \sigma_{i}\right\rangle$, with $p_{i}$ and $\sigma_{i}$ denoting the individual (free) fourmomenta and spin projections, respectively. In point form, instead of working with the usual three-body states in eq. (4), one introduces so-called velocity states. They can be constructed by applying a specific Lorentz boost $B(v)$ to the free three-body states $\left|k_{1}, \mu_{1} ; k_{2}, \mu_{2} ; k_{3}, \mu_{3}\right\rangle$ in the centre-of-momentum frame (for which $\sum_{i} \vec{k}_{i}=0$ ):

$$
\left|v ; \vec{k}_{1}, \mu_{1} ; \vec{k}_{2}, \mu_{2} ; \vec{k}_{3}, \mu_{3}\right\rangle=U_{B(v)}\left|k_{1}, \mu_{1} ; k_{2}, \mu_{2} ; k_{3}, \mu_{3}\right\rangle=\prod_{i=1}^{3} \sum_{\sigma_{i}} D_{\sigma_{i} \mu_{i}}^{\frac{1}{2}}\left[R_{W}\left(k_{i}, B(v)\right)\right]\left|p_{1}, \sigma_{1} ; p_{2}, \sigma_{2} ; p_{3}, \sigma_{3}\right\rangle .
$$

These velocity states also span the whole Hilbert space $\mathcal{H}$. They have the important advantage that under general Lorentz transformations the occurring Wigner $D$-functions are the same for all three particles and the individual momenta are all rotated by the same amount (what is not the case for the three-particle states of eq. (4)). Of course, the practical calculations are facilitated a lot by expressing the baryon mass eigenstates $\left|\Psi_{B}\right\rangle$ in the velocity-state representation

$$
\left\langle v ; \vec{k}_{1}, \mu_{1} ; \vec{k}_{2}, \mu_{2} ; \vec{k}_{3}, \mu_{3} \mid v_{B}, M_{B}, J, \Sigma\right\rangle \sim \delta^{3}\left(\vec{v}-\vec{v}_{B}\right) \Psi_{M_{B} J \Sigma}\left(\vec{k}_{1}, \mu_{1} ; \vec{k}_{2}, \mu_{2} ; \vec{k}_{3}, \mu_{3}\right)
$$

where $\vec{v}$ and $\vec{v}_{B}$ are the total three-velocities of the bra and ket states, respectively.

\section{Invariant Nucleon Form Factors}

For the elastic electromagnetic form factors one has to compute matrix elements of the current operator $\hat{J}^{\mu}(x)$ between the baryon states. The electromagnetic current operator itself is an irreducible tensor operator of the Poincaré 
group. One can apply a generalized Wigner-Eckart theorem and decompose the matrix elements into Clebsch-Gordan coefficients times reduced matrix elements, which are the invariant form factors [12]. Due to the covariance properties of the matrix elements one can use any reference frame and proceed with the calculation. We choose the usual Breit frame, where the Clebsch-Gordan coefficients are unity. The elastic invariant form factors are then given by the matrix elements of the current operator $\hat{\mathcal{J}}^{\mu}(0)$ between incoming and outgoing baryon states boosted to the (standard) Breit frame

$$
2 M_{B} F_{\Sigma^{\prime} \Sigma}^{\mu}\left(Q^{2}\right)=\left\langle v_{B}^{\prime}, M_{B}, J, \Sigma^{\prime}\left|\hat{\mathcal{J}}^{\mu}(0)\right| v_{B}, M_{B}, J, \Sigma\right\rangle
$$

The invariant momentum transfer $q^{2}=-Q^{2}$ along the $z$-axis is defined as the difference between the final and the initial four-momenta of the baryon, $P^{\mu \prime}=M_{B} v_{B}^{\prime}$ and $P^{\mu}=M_{B} v_{B}$, respectively,

$$
q^{\mu}=(0,0,0, Q)=P^{\mu \prime}-P^{\mu}
$$

where $v_{B}$ and $v_{B}^{\prime}$ are the initial and final baryon four-velocities.

The invariant form factors are related to the electric and magnetic Sachs form factors $G_{E}$ and $G_{M}$, respectively. In the Breit frame one has for spin- $\frac{1}{2}$ baryons

$$
G_{E}=F_{\frac{1}{2} \frac{1}{2}}^{\mu=0}, \quad G_{M}=\frac{2 M_{B}}{Q} F_{\frac{1}{2}-\frac{1}{2}}^{\mu=1}
$$

and for spin- $\frac{3}{2}$ baryons [15, 16]

$$
G_{E}=\frac{1}{2}\left(F_{\frac{1}{2} \frac{1}{2}}^{\mu=0}+F_{\frac{3}{2} \frac{3}{2}}^{\mu=0}\right), \quad G_{M}=\frac{6}{5} \frac{M_{B}}{Q}\left(F_{\frac{1}{2}-\frac{1}{2}}^{\mu=1}+\sqrt{3} F_{\frac{3}{2} \frac{1}{2}}^{\mu=1}\right) .
$$

\section{Point Form Current Model}

In case of a relativistic three-body system, the matrix elements in eq. (7) cannot be calculated with the full structure of the electromagnetic current operator. Rather one has to resort to simplifications. For the three-body electromagnetic current operator we therefore assume a spectator model, i.e. only a single quark directly couples to the virtual photon while the other two act as spectators

$$
\begin{aligned}
& \left\langle p_{1}^{\prime}, \sigma_{1}^{\prime} ; p_{2}^{\prime}, \sigma_{2}^{\prime} ; p_{3}^{\prime}, \sigma_{3}^{\prime}\left|\hat{\mathcal{J}}^{\mu}(0)\right| p_{1}, \sigma_{1} ; p_{2}, \sigma_{2} ; p_{3}, \sigma_{3}\right\rangle \\
& \quad=3\left(\frac{M_{B}^{2}}{M_{\text {free }} M_{\text {free }}^{\prime}}\right)^{\frac{3}{2}} \delta^{3}\left(\vec{p}_{2}^{\prime}-\vec{p}_{2}\right) \delta^{3}\left(\vec{p}_{3}^{\prime}-\vec{p}_{3}\right) \times \delta_{\sigma_{2}^{\prime} \sigma_{2}} \delta_{\sigma_{3}^{\prime} \sigma_{3}} 2 E_{2} 2 E_{3}\left\langle p_{1}^{\prime}, \sigma_{1}^{\prime}\left|\hat{\mathcal{J}}_{[1]}^{\mu}(0)\right| p_{1}, \sigma_{1}\right\rangle
\end{aligned}
$$

where $E_{i}=\sqrt{\vec{p}_{i}^{2}+m_{i}^{2}}(i=2,3)$ are the energies of the spectator quarks. The single-particle current is taken in the usual form for a pointlike Dirac particle with charge $e_{n}(n=1,2,3)$

$$
\left\langle p_{n}^{\prime}, \sigma_{n}^{\prime}\left|\hat{\mathcal{J}}_{[1]}^{\mu}(0)\right| p_{n}, \sigma_{n}\right\rangle=e_{n} \bar{u}\left(p_{n}^{\prime}, \sigma_{n}^{\prime}\right) \gamma^{\mu} u\left(p_{n}, \sigma_{n}\right)
$$

where the quark spinor can be expressed in terms of the two-component Pauli spinor $\chi$ in the following way

$$
u(p, \sigma)=\sqrt{E+m}\left(\begin{array}{c}
\chi \\
\vec{\sigma} \cdot \vec{p} \\
E+m
\end{array}\right) .
$$

Eqs. (11) and (12) define the so-called point-form spectator approximation (PFSA). It should be noted that the PFSA current model does not represent a pure one-body operator. Even though the virtual photon couples only to a single quark, also the spectator quarks participate in the process. The whole baryon experiences a boost due to the total momentum transfer $q^{2}$, which is then shared by all quarks. Only a part of it is transferred to the struck quark, namely,

$$
\tilde{q}^{2}=\left(p_{n}^{\prime}-p_{n}\right)^{2}=\left[B\left(v_{B}^{\prime}\right) k_{n}^{\prime}-B\left(v_{B}\right) k_{n}\right]^{2}
$$

Furthermore, since in point form the momenta are affected by interactions, the PFSA current rather represents an effective dynamical three-body current. 
In PFSA, the matrix elements of the current operator are finally given by the multiple integral

$$
\begin{aligned}
& F_{\Sigma^{\prime} \Sigma}^{\mu}\left(Q^{2}\right)=3 \int d \vec{k}_{2} d \vec{k}_{3} d \vec{k}_{2}^{\prime} d \vec{k}_{3}^{\prime}\left(\frac{M_{B}^{2}}{M_{f r e e} M_{\text {free }}^{\prime}}\right)^{\frac{3}{2}} \sqrt{\frac{\omega_{2}^{\prime} \omega_{3}^{\prime}}{\omega_{2} \omega_{3}}} \delta^{3}\left[k_{2}^{\prime}-B^{-1}\left(v_{B}^{\prime}\right) B\left(v_{B}\right) k_{2}\right] \\
& \times \delta^{3}\left[k_{3}^{\prime}-B^{-1}\left(v_{B}^{\prime}\right) B\left(v_{B}\right) k_{3}\right] \Psi_{M_{B} J \Sigma^{\prime}}^{*}\left(\vec{k}_{1}^{\prime}, \mu_{1}^{\prime} ; \vec{k}_{2}^{\prime}, \mu_{2}^{\prime} ; \vec{k}_{3}^{\prime}, \mu_{3}^{\prime}\right) D_{\sigma_{1}^{\prime} \mu_{1}^{\prime}}^{1 / 2}{ }^{*}\left[R_{W}\left(k_{1}^{\prime}, B\left(v_{B}^{\prime}\right)\right)\right] \\
& \times \frac{1}{2 \sqrt{\omega_{1} \omega_{1}^{\prime}}}\left\langle p_{1}^{\prime}, \sigma_{1}^{\prime}\left|\hat{\mathcal{J}}_{[1]}^{\mu}(0)\right| p_{1}, \sigma_{1}\right\rangle D_{\sigma_{1} \mu_{1}}^{1 / 2}\left[R_{W}\left(k_{1}, B\left(v_{B}\right)\right)\right] \Psi_{M_{B} J \Sigma}\left(\vec{k}_{1}, \mu_{1} ; \vec{k}_{2}, \mu_{2} ; \vec{k}_{3}, \mu_{3}\right) \\
& \times D_{\mu_{2}^{\prime} \mu_{2}}^{1 / 2}\left[R_{W}\left(k_{2}, B^{-1}\left(v_{B}^{\prime}\right) B\left(v_{B}\right)\right)\right] D_{\mu_{3}^{\prime} \mu_{3}}^{1 / 2}\left[R_{W}\left(k_{3}, B^{-1}\left(v_{B}^{\prime}\right) B\left(v_{B}\right)\right)\right],
\end{aligned}
$$

where $\omega_{n}=\sqrt{\vec{k}_{n}^{2}+m_{n}^{2}}$ are the single-quark energies in the baryon centre-of-momentum frame.

\section{Charge Radii and Magnetic Moments}

The baryon charge radii $r_{c h}^{2}$ and magnetic moments $\mu$ can be calculated from the electric and magnetic Sachs form factors of eqs. (9) - (10) in the limit $Q^{2} \rightarrow 0$ :

$$
r_{c h}^{2} \equiv-\left.6 \frac{d G_{E}}{d\left(Q^{2}\right)}\right|_{Q^{2}=0}, \quad \mu \equiv G_{M}\left(Q^{2}=0\right)
$$

\section{RESULTS AND DISCUSSION}

The predictions of the GBE CQM for the charge radii and magnetic moments are given in Tables 【 and [I] for all octet and decuplet baryons, where experimental data are available. The quoted PFSA results are manifestly covariant and they are immediately found in good or reasonable agreement with experiment in all cases. Their quality is similar to the one of the nucleon electroweak form factors obtained before [9, 10, 13. This is remarkable because the results represent direct predictions obtained just with the quark model eigenstates, without introducing any further phenomenological parametrisations such as quark form factors etc.

We have also studied the influence of relativity on the results. First we compare to the calculations in nonrelativistic impulse approximation (NRIA), see Tables [II and [V] For the charge radii the shortcomings of the nonrelativistic approach are immediately evident. Considerable effects are caused by both the relativistic current operator (cf. columns 1 and 2 of Table III) and the relativistic boosts (cf. column 3 of Table III). Only the covariant results (last column of Table III) turn out to be reasonable and compare well with experimental data, whenever such a comparison is possible (see Table 【).

For the magnetic moments seemingly good results are obtained with the NRIA, especially in case of the nucleons and some other octet baryons. However, this has to be considered as accidental. Already in the decuplet baryons, the influence of relativity becomes rather large. From the relativistic (PFSA) calculation, which employs a relativistic current operator and also includes boost effects, one learns that both of these ingredients are necessary in order to produce a reasonable prediction in concordance with experiment (cf. the last three columns in Table IV]. In other words: In the covariant calculation, relativistic effects (both from the current and the boosts) appear even though the magnetic moments are observables at $Q^{2}=0$. In case a nonrelativistic current is used, Lorentz boosts have essentially no effect, since they enter only in higher orders of $Q$. As a consequence the deviations from the covariant results may become considerable (up to about $30 \%$, for example, in the case of $\Delta^{+}$).

In summary, it appears evident that, in order to reach consistent results, any calculation of both the charge radii and the magnetic moments must be performed in a fully relativistic manner, employing a relativistic current operator as well as including Lorentz boosts. This is true even though we deal here with observables in the limit $Q^{2} \rightarrow 0$. The point-form approach appears as a reasonable framework to obtain covariant predictions for charge radii as well as magnetic moments.

[1] C. Alexandrou, P. de Forcrand, and A. Tsapalis, Phys. Rev. D 66, 094503 (2002). 
[2] M. Goeckeler et al., arXiv:hep-ph/010810 (2001).

[3] M. K. Jones, Nucl. Phys. A 699, 124c (2002).

[4] G. S. Bali, in: NSTAR 2002, (Proceedings of the Workshop on the Physics of Excited Nucleons, Pittsburgh) (World Scientific, Singapore, 2003).

[5] L. Y. Glozman, W. Plessas, K. Varga, and R. F. Wagenbrunn, Phys. Rev. D 58, 094030 (1998).

[6] L. Y. Glozman and D. O. Riska, Phys. Rep. 268, 263 (1996).

[7] L. Y. Glozman et al., Phys. Rev. C 57, 3406 (1998).

[8] B. D. Keister and W. N. Polyzou, Adv. Nucl. Phys. 20, 225 (1991).

[9] R. F. Wagenbrunn et al., Phys. Lett. B 511, 33 (2001).

[10] L. Y. Glozman et al., Phys. Lett. B 516, 183 (2001).

[11] P. A. M. Dirac, Rev. Mod. Phys. 21, 392 (1949).

[12] W. H. Klink, Phys. Rev. C 58, 3587 (1998).

[13] S. Boffi et al., Eur. Phys. J. A 14, 17 (2002).

[14] B. Bakamjian and L. H. Thomas, Phys. Rev. 92, 1300 (1953).

[15] I. L. Durand, P. DeCelles, and R. Marr, Phys. Rev. 126, 1882 (1962).

[16] N. R. Nath and H. J. Weber, Phys. Rev. D 6, 1975 (1972).

[17] K. Hagiwara et al., Phys. Rev. D 66, 010001 (2002).

[18] M. Kotulla et al., Acta Phys. Polon. B 33, 957 (2002).

TABLE I: PFSA predictions of the GBE CQM for baryon charge radii $r_{c h}^{2}\left[\mathrm{fm}^{2}\right]$ in comparison to experiment 17].

\begin{tabular}{lrr}
\hline \hline Baryon & GBE CQM & Experimental \\
& PFSA & Data \\
\hline$p$ & 0.82 & $0.757 \pm 0.014$ \\
$n$ & -0.13 & $-0.1161 \pm 0.0022$ \\
$\Sigma^{-}$ & 0.72 & $0.61 \pm 0.12 \pm 0.09$ \\
\hline \hline
\end{tabular}

TABLE II: PFSA predictions of the GBE CQM for baryon magnetic moments (in n.m.) compared to experiment [17].

\begin{tabular}{lrr}
\hline \hline Baryon & GBE CQM & Experimental \\
& PFSA & 2.7928 \\
\hline$p$ & 2.70 & -1.9130 \\
$n$ & -1.70 & $-0.613 \pm 0.004$ \\
$\Lambda$ & -0.65 & $2.458 \pm 0.010$ \\
$\Sigma^{+}$ & 2.35 & $-1.160 \pm 0.025$ \\
$\Sigma^{-}$ & -0.92 & $-1.250 \pm 0.014$ \\
$\Xi^{0}$ & -1.24 & $-0.6507 \pm 0.0025$ \\
$\Xi^{-}$ & -0.68 & $2.7_{-1.3}^{+1.0} \pm 1.5 \pm 3^{a}$ \\
$\Delta^{+}$ & 2.08 & $3.7-7.5$ \\
$\Delta^{++}$ & 4.17 & $-2.020 \pm 0.05$ \\
$\Omega^{-}$ & -1.59 & -020 \\
\hline \hline
\end{tabular}

${ }^{a}$ This result is taken from ref. [18]. 
TABLE III: Results for charge radii $\left(\right.$ in $\mathrm{fm}^{2}$ ) of all octet and decuplet baryons in NRIA, a calculation using the relativistic current and no boosts, a calculation using the nonrelativistic current with boosts included, and in PFSA.

\begin{tabular}{|c|c|c|c|c|}
\hline Baryon & NRIA & $\begin{array}{r}\mathrm{RC} w / \mathrm{o} \\
\text { Boosts }\end{array}$ & $\begin{array}{r}\mathrm{NRC}+ \\
\text { Boosts }\end{array}$ & PFSA \\
\hline $\bar{p}$ & 0.10 & 0.18 & 0.58 & 0.82 \\
\hline$n$ & -0.01 & -0.01 & -0.09 & -0.13 \\
\hline$\Lambda$ & 0.01 & 0.02 & 0.01 & 0.03 \\
\hline$\Sigma^{0}$ & 0.02 & 0.03 & 0.14 & 0.20 \\
\hline$\Sigma^{+}$ & 0.12 & 0.21 & 0.77 & 1.13 \\
\hline$\Sigma^{-}$ & 0.09 & 0.16 & 0.50 & 0.72 \\
\hline$\Xi^{0}$ & 0.01 & 0.03 & -0.17 & -0.19 \\
\hline$\Xi^{-}$ & 0.10 & 0.16 & 0.41 & 0.54 \\
\hline$\Sigma^{* 0}$ & 0.02 & 0.03 & 0.01 & 0.03 \\
\hline$\Sigma^{*+}$ & 0.20 & 0.32 & 0.32 & 0.42 \\
\hline$\Sigma^{*-}$ & 0.16 & 0.25 & 0.29 & 0.37 \\
\hline$\Delta^{+}$ & 0.15 & 0.25 & 0.32 & 0.43 \\
\hline$\Delta^{0}$ & 0.00 & 0.00 & 0.00 & 0.00 \\
\hline$\Delta^{++}$ & 0.15 & 0.25 & 0.32 & 0.43 \\
\hline$\Delta^{-}$ & 0.15 & 0.25 & 0.32 & 0.43 \\
\hline$\Xi^{* 0}$ & 0.04 & 0.07 & 0.03 & 0.06 \\
\hline$\Xi^{*-}$ & 0.16 & 0.24 & 0.26 & 0.33 \\
\hline$\Omega^{-}$ & 0.16 & 0.22 & 0.24 & 0.29 \\
\hline
\end{tabular}

TABLE IV: Results for magnetic moments (in n.m.) of all octet and decuplet baryons in NRIA, a calculation using the relativistic current and no boosts, a calculation using the nonrelativistic current with boosts included, and in PFSA.

\begin{tabular}{|c|c|c|c|c|}
\hline Baryon & NRIA & $\begin{array}{r}\mathrm{RC} \mathrm{w} / \mathrm{o} \\
\text { Boosts }\end{array}$ & $\begin{array}{r}\text { NRC }+ \\
\text { Boosts }\end{array}$ & PFSA \\
\hline$p$ & 2.74 & 1.31 & 2.74 & 2.70 \\
\hline$n$ & -1.82 & -0.85 & -1.82 & -1.70 \\
\hline$\Lambda$ & -0.61 & -0.37 & -0.61 & -0.65 \\
\hline$\Sigma^{0}$ & 0.81 & 0.39 & 0.81 & 0.72 \\
\hline$\Sigma^{+}$ & 2.63 & 1.25 & 2.62 & 2.35 \\
\hline$\Sigma^{-}$ & -1.01 & -0.46 & -1.01 & -0.92 \\
\hline$\Xi^{0}$ & -1.40 & -0.79 & -1.40 & -1.24 \\
\hline$\Xi^{-}$ & -0.53 & -0.38 & -0.53 & -0.68 \\
\hline$\Sigma^{* 0}$ & 0.29 & 0.08 & 0.29 & 0.09 \\
\hline$\Sigma^{*+}$ & 3.06 & 1.62 & 3.06 & 2.07 \\
\hline$\Sigma^{*-}$ & -2.47 & -1.45 & -2.47 & -1.89 \\
\hline$\Delta^{+}$ & 2.76 & 1.51 & 2.76 & 2.08 \\
\hline$\Delta^{0}$ & 0.00 & 0.00 & 0.00 & 0.00 \\
\hline$\Delta^{++}$ & 5.52 & 3.03 & 5.52 & 4.17 \\
\hline$\Delta^{-}$ & -2.76 & -1.51 & -2.76 & -2.08 \\
\hline$\Xi^{* 0}$ & 0.59 & 0.17 & 0.59 & 0.18 \\
\hline$\Xi^{*-}$ & -2.17 & -1.38 & -2.17 & -1.73 \\
\hline$\Omega^{-}$ & -1.88 & -1.29 & -1.88 & -1.59 \\
\hline
\end{tabular}

